\title{
GEOGRAPHICAL DISTRIBUTION OF MEMBERS UNITED STATES AND POSSESSIONS
}

\begin{tabular}{|c|c|c|c|}
\hline & $\begin{array}{l}\text { F. Irwin } \\
\text { R. D. James } \\
\text { D. N. Lehmer } \\
\text { Lenzen } \\
\text { Leuschner } \\
\text { S. H. Levy } \\
\text { Lewy } \\
\text { Morrey } \\
\text { Mowbray } \\
\text { Noble } \\
\text { T. M. Putnam } \\
\text { Sperry } \\
\text { Sugar } \\
\text { Swinford } \\
\text { J. M. Thompson } \\
\text { A. R. Williams } \\
\text { Wong } \\
\text { Brawley } \\
\text { Pemberton } \\
\text { Claremont } \\
\text { H. J. Hamilton } \\
\text { Jaeger } \\
\text { Compton } \\
\text { Duncan } \\
\text { Davis } \\
\text { Roessler } \\
\text { Fresno } \\
\text { F. R. Morris } \\
\text { Glendale } \\
\text { Harrington } \\
\text { La Jolla } \\
\text { G. F. McEwen } \\
\text { Los Angeles } \\
\text { Collier } \\
\text { Koch } \\
\text { Los Angeles Jun- } \\
\text { ior College } \\
\text { Urner } \\
\text { University of } \\
\text { California at } \\
\text { Los Angeles } \\
\text { C. Bell } \\
\text { Daus } \\
\text { Glazier } \\
\text { E. R. Hedrick } \\
\text { Hestenes } \\
\text { G. H. Hunt } \\
\text { G. James } \\
\text { Sherwood } \\
\text { W. M. Whyburn } \\
\text { Worthington } \\
\text { Zorn } \\
\text { University of } \\
\text { Southern Cali- } \\
\text { fornia } \\
\text { L. D. Ames } \\
\text { Steed } \\
\text { Ste }\end{array}$ & $\begin{array}{l}\text { Pasadena } \\
\text { A. E. Taylor } \\
\text { California Insti- } \\
\text { tute of Technol- } \\
\text { ogy } \\
\text { Bateman } \\
\text { E. T. Bell } \\
\text { Birchby } \\
\text { M. Mason } \\
\text { Michal } \\
\text { Tolman } \\
\text { Van Buskirk } \\
\text { M. Ward } \\
\text { Wear } \\
\text { Redlands } \\
\text { O. W. Albert } \\
\text { San Diego } \\
\text { Klauber } \\
\text { San Francisco } \\
\text { Haley } \\
\text { McCarty } \\
\text { San Jose } \\
\text { Heaslet } \\
\text { Santa Ana } \\
\text { Whiting } \\
\text { Santa Monica } \\
\text { L. J. Adams } \\
\text { Stanford Univer- } \\
\text { sity } \\
\text { C. L. Alsberg } \\
\text { H. M. Bacon } \\
\text { Blichfeldt } \\
\text { W. F. Durand } \\
\text { Harrold } \\
\text { Hoskins } \\
\text { Luther } \\
\text { W. A. Manning } \\
\text { Uspensky } \\
\text { Stockton } \\
\text { Corbin } \\
\text { CoLoRADo } \\
\text { Boulder } \\
\text { Beaty } \\
\text { Britton } \\
\text { DeLong } \\
\text { Hutchinson } \\
\text { Kempner } \\
\text { Kendall } \\
\text { McMaster } \\
\text { Colorado Springs } \\
\text { Lovitt } \\
\text { Rawles } \\
\text { Roos } \\
\text { Sisam } \\
\text { Denver } \\
\text { Powers } \\
\text { Sabin } \\
\text { Salo }\end{array}$ & 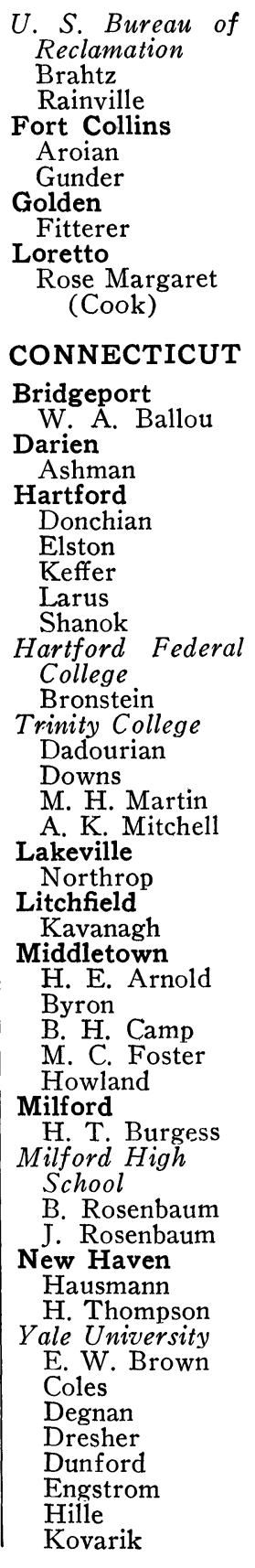 \\
\hline
\end{tabular}


Longley

E. J. Miles

Ore

Page

D. T. Perkins

Raudenbush

Riblet

M. S. Robertson

P. F. Smith

Tracey

Uhler

Whittemore

W. A. Wilson

Wrench

New London

Connecticut Col-

lege

J. W. Bower

Leib

U.S. Coast Guard Academy

Dimick

Storrs

Cheney

C. H.W. Sedgewick

R. W. Sedgewick

Washington

Gurney

DELAWARE

Cannon

Cannon

Claymont

Sromovsky

Laurel J. R. K. Stauffer

Newark

Harter

McDougle

C. J. Rees

DISTRICT OF COLUMBIA

Washington

H. S. Adams

Bradt

Cromwell

H. K. Cummings

Dabney

Edmonston

Greville

H. B. Hedrick

Kullback

Nagle

Perlo

Sasuly

Tyler

Van Orstrand

American University

Aitchison

Catholic University of America
Finan

Landry

Ramler

J. N. Rice

Georgetown University

Sohon

George Washing-

ton University

Johnston

Mears

J. H. Taylor

Weida

Howard

sity

G. M. Jones

Woodard

Miner Teachers

College

Haynes

Trinity College Thomas Marie (Maloney)

$U$. S. Coast and Geodetic Survey

O. S. Adams

A. Blake

Darling

Lambert

U. S. Navy De-

partment

Goldberg

Larrivee

Steinert

Wertheimer

$U$. S. Weather

Bureau

W. J. Humphreys

Woolard

\section{FLORIDA}

Deland

Faulkner

Gainesville

U. P. Davis

Dostal

Kokomoor

Kusner

Phipps

Pirenian

Quade

Simpson

W. H. Wilson

Jacksonville

H. Rosser

Lakeland

Reinsch

Winter Park

Hutchings

\section{GEORGIA}

Atlanta

Georgia School of
D. H. Ballou

J. E. Boyd

Fulmer

Hefner

Sewell

D. M. Smith

Steen

Spelman College

Caldwell

Carrollton

M. E. Howell

Lang

Decatur

H. A. Robinson

Macon

Wade

Milledgeville

S. L. Nelson

Mt. Berry

S. H. Cook

HAWAII

Honolulu

Castle

Yap

IDAHO

Boise

A. B. Carson

Caldwell

Rankin

Moscow

Bender

E. Taylor

ILLINOIS

Bloomington

M. Hunt

Carbondale

Mayor

Chicago

F. W. Boyce

C. P. Brady

E. R. Carter

Fordney

Hornberger

Levinson

F. R. Moulton

Paul

A. R. Schweitzer

B. Smith

Armour Institute

of Technology

Bibb

D. F. Campbell

W. M. Davis

Krathwohl

Oldenburger

G. C. Webber

Central Y.M.C.A College

J. E. Davis

Gore
DePaul University Corliss

Loyola University Gerst

O'Mara

Tordella

Mundelein College Mary Sylvester

St. Francis Xavier College

E. C. Pixley

University of Chicago

A. A. Albert

Barnard

Bartky

Bliss

Denbow

Dickson

Everett

Goldstine

L. M. Graves

R. Hull

Jacobson

E. P. Lane

Logsdon

Lunn

MacMillan

Moscovitch

Rasmusen

Reid

Sanger

Schultz

Slaught

M. F. Smiley

Valentine

J. W. A. Young

Woodrow Wilson

Junior College

Kinney

DeKalb

Hellmich

Eureka

Newson

Evanston

D. R. Curtiss

H. L. Garabedian

Griffiths

Holgate

E. J. Moulton

Randels

Simmons

Wall

F. E. Wood

Galesburg

Sellew

R. C. Stephens

Hinsdale

F. H. Murray

Joliet

F. C. Smith

Lake Forest

Curtis 


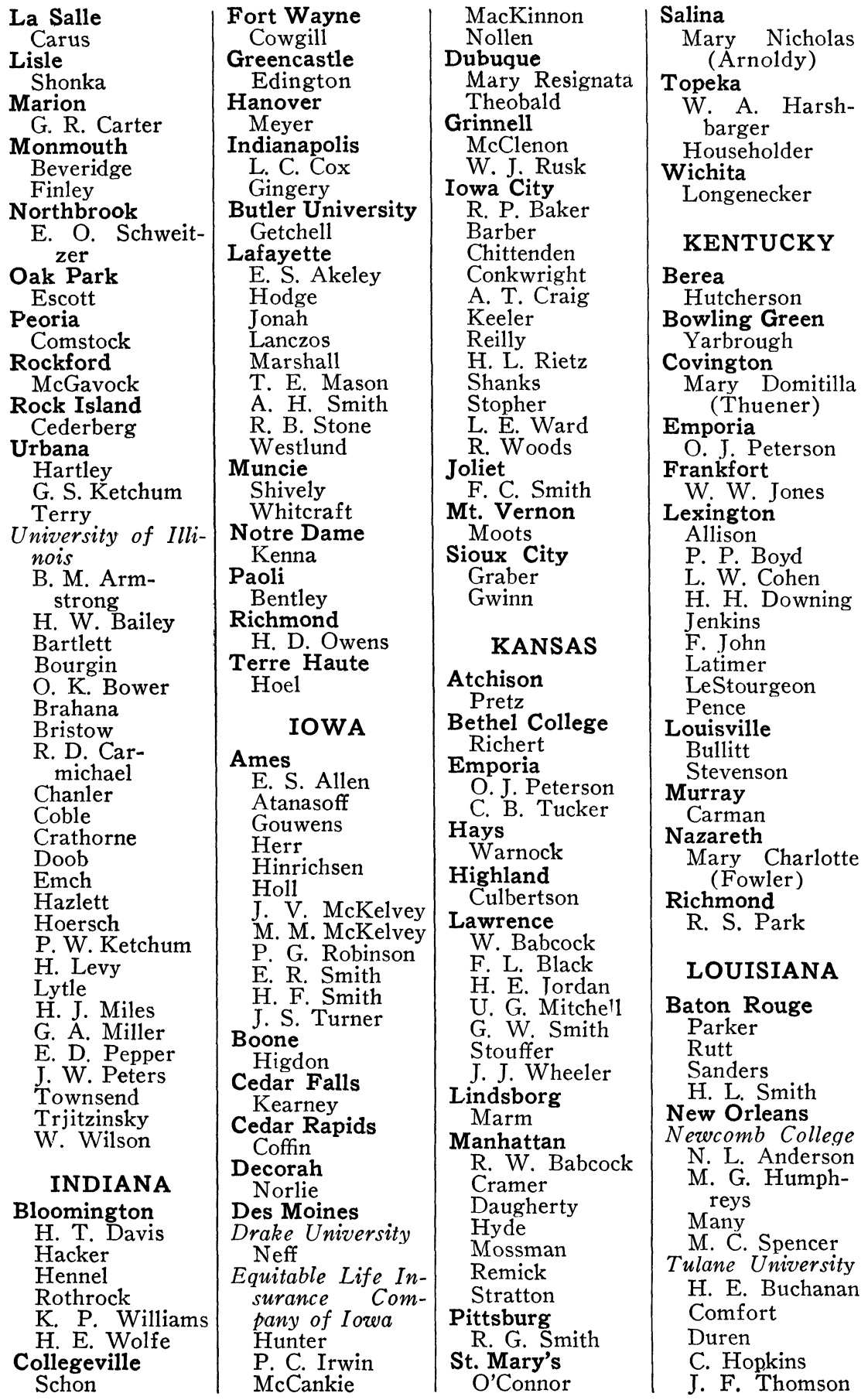




\begin{tabular}{|c|c|c|c|}
\hline $\begin{array}{l}\text { Brunswick } \\
\text { Hammond } \\
\text { Holmes } \\
\text { Korgen } \\
\text { W. A. Moody } \\
\text { Orono } \\
\text { Bryan } \\
\text { S. H. Kimball } \\
\text { Waterville } \\
\text { Schoenberg } \\
\text { MARYLAND } \\
\text { Aberdeen } \\
\text { Dederick } \\
\text { Annapolis } \\
\text { Ball } \\
\text { Bramble } \\
\text { Capron } \\
\text { R. Church } \\
\text { Clements } \\
\text { A. E. Currier } \\
\text { Kells } \\
\text { Littauer } \\
\text { T. W. Moore } \\
\text { Rawlins } \\
\text { Root } \\
\text { Scarborough } \\
\text { L.T. Wilson } \\
\text { Baltimore } \\
\text { Dawkins } \\
\text { Dorroh } \\
\text { L. B. Robinson } \\
\text { Sutton } \\
\text { Goucher College } \\
\text { C. L. Bacon } \\
\text { F. P. Lewis } \\
\text { Torrey } \\
\text { Johns Hopkins } \\
\text { University } \\
\text { A. Cohen } \\
\text { J. H. Curtiss } \\
\text { R. H. Fox } \\
\text { Harry } \\
\text { Haviland } \\
\text { van Kampen } \\
\text { Kershner } \\
\text { F. Morley } \\
\text { Morrill } \\
\text { Murnaghan } \\
\text { L. J. Reed } \\
\text { Trott } \\
\text { Turpin } \\
\text { J. Williamson } \\
\text { Wintner } \\
\text { Zariski } \\
\text { Cheltenham } \\
\text { Hartwell } \\
\text { Chestertown } \\
\text { W. J. Robinson } \\
\text { College Park } \\
\text { Dantzig } \\
\text { Frederick } \\
\text { R. G. Mason } \\
\text { Hation }\end{array}$ & $\begin{array}{l}\text { Westminster } \\
\text { Spicer } \\
\text { MASSACHU- } \\
\text { SETTS } \\
\text { Amherst } \\
\text { B. L. Brown } \\
\text { C. W. Cobb } \\
\text { Esty } \\
\text { Sprague } \\
\text { Belmont } \\
\text { C. W. Franklin } \\
\text { R. R. Struik } \\
\text { Boston } \\
\text { Cleary } \\
\text { Dysart } \\
\text { A. B. Gould } \\
\text { L. A. McCoy } \\
\text { Pousland } \\
\text { Rines } \\
\text { Boston University } \\
\text { Bruce } \\
\text { Mode } \\
\text { Graduate School } \\
\text { of Business Ad- } \\
\text { ministration, } \\
\text { Harvard Uni- } \\
\text { versity } \\
\text { T. H. Brown } \\
\text { Leavens } \\
\text { Harvard } \\
\text { of Public Health } \\
\text { E. B. Wilson } \\
\text { John Hancock Mu- } \\
\text { tual Life Insur- } \\
\text { ance Company } \\
\text { Abbott } \\
\text { H. A. Garabe- } \\
\text { dian } \\
\text { Grout } \\
\text { Howe } \\
\text { Vatter } \\
\text { Northeastern Uni- } \\
\text { versity } \\
\text { E. E. Haskins } \\
\text { Spear } \\
\text { Simmons College } \\
\text { Hemenway } \\
\text { Brockton } \\
\text { N. Kaplan } \\
\text { Brookline } \\
\text { Groat } \\
\text { A. L. Miller } \\
\text { Cambridge } \\
\text { Galbraith } \\
\text { Korzybski } \\
\text { E. W. Wilson } \\
\text { Harvard Univer- } \\
\text { sity } \\
\text { Ahlfors } \\
\text { Andrews } \\
\text { Beatley } \\
\text { G. Birkhoff } \\
\text { G. D. Birkhoff }\end{array}$ & 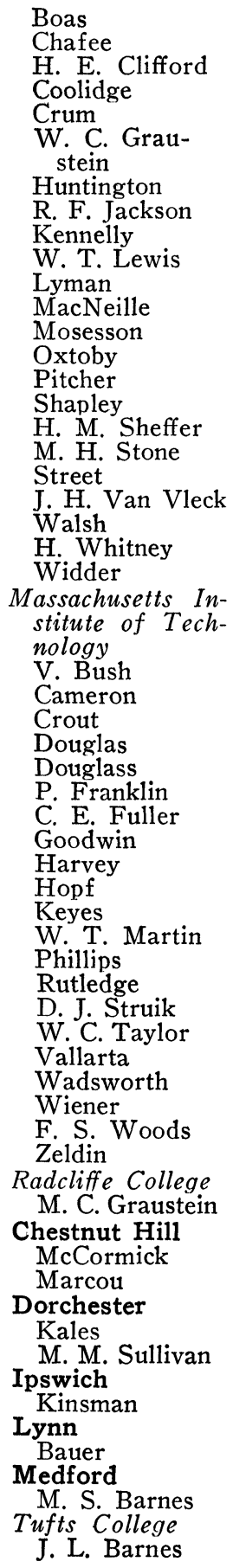 & 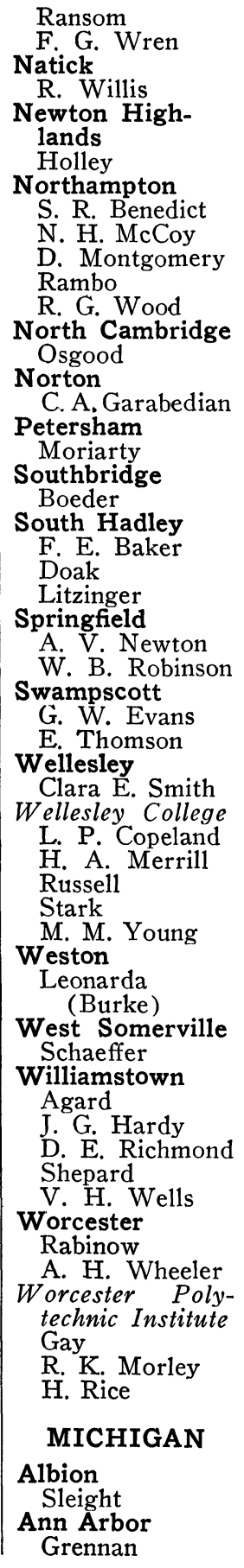 \\
\hline
\end{tabular}




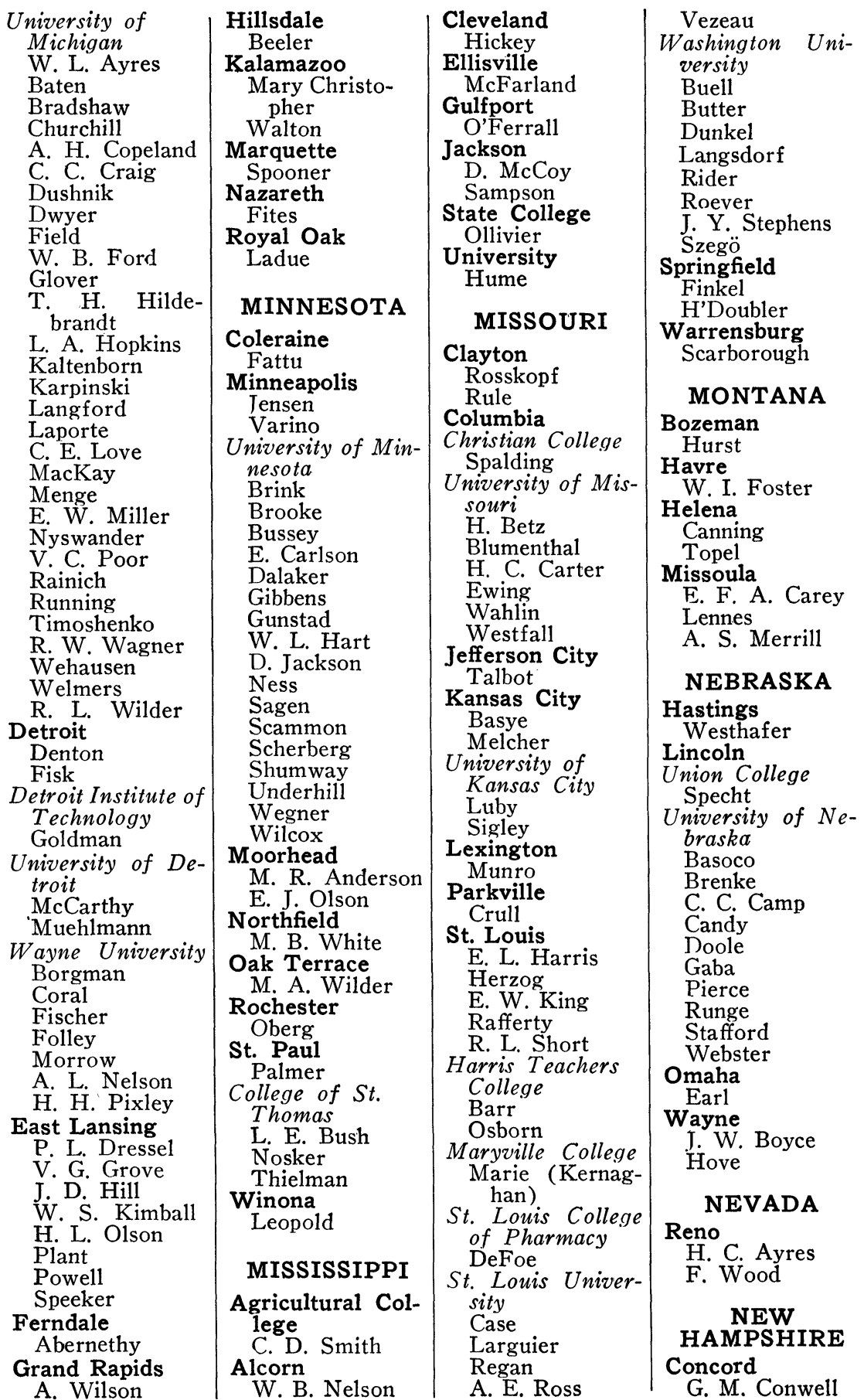




\begin{tabular}{|c|c|c|c|}
\hline $\begin{array}{l}\text { ell } \\
\text { er } \\
\text { Ban } \\
\text { gan } \\
\text { uth College } \\
\text { e Brown } \\
\text { yth } \\
\text {. Haskins } \\
\text { ewson } \\
\text { ow Perkins } \\
\text { obinson } \\
\text { rman } \\
\text { Wilder }\end{array}$ & $\begin{array}{l}\text { Walter } \\
\text { Rutgers Univer- } \\
\text { sity } \\
\text { Brasefield } \\
\text { Bunyan } \\
\text { H. S. Grant } \\
\text { R. Morris } \\
\text { Starke } \\
\text { Orange } \\
\text { Strock } \\
\text { Perth Amboy } \\
\text { Fullerton } \\
\text { Princeton } \\
\text { Burton } \\
\text { Flood } \\
\text { Parke } \\
\text { R. C. Stauffer } \\
\text { Wallman } \\
\text { Institute for Ad- } \\
\text { vanced Study } \\
\text { Alexander } \\
\text { Baer } \\
\text { A. H. Clifford } \\
\text { Comenetz } \\
\text { Fialkow } \\
\text { M. Hall } \\
\text { M. Kline } \\
\text { R. S. Martin } \\
\text { M. Morse } \\
\text { Myers } \\
\text { von Neumann } \\
\text { G. B. Price } \\
\text { M. Richardson } \\
\text { Tompkins } \\
\text { Veblen } \\
\text { Weyl } \\
\text { Zippin } \\
\text { Princeton Univer- } \\
\text { sity } \\
\text { E.P. Adams } \\
\text { Allendoerfer } \\
\text { Bochner } \\
\text { Bohnenblust } \\
\text { A. Church } \\
\text { Dribin } \\
\text { Eisenhart } \\
\text { Gillespie } \\
\text { Knebelman } \\
\text { Lefschetz } \\
\text { H. P. Robertson } \\
\text { Steenrod } \\
\text { T. Y. Thomas } \\
\text { Titt } \\
\text { A. W. Tucker } \\
\text { Wedderburn } \\
\text { Wigner } \\
\text { Wilks } \\
\text { Willson } \\
\text { Di1 }\end{array}$ & $\begin{array}{l}\text { Shuster } \\
\text { Upper Montclair } \\
\text { G. A. Campbell } \\
\text { Nezw Jersey State } \\
\text { Teachers Col- } \\
\text { lege } \\
\text { D. R. Davis } \\
\text { Fehr } \\
\text { E. H. C. Hilde- } \\
\text { brandt } \\
\text { Mallory } \\
\text { West Orange } \\
\text { Edison } \\
\text { NEW MEXICO } \\
\text { Albuquerque } \\
\text { Barnhart } \\
\text { Newsom } \\
\text { Las Vegas } \\
\text { Rodgers } \\
\text { Roswell } \\
\text { Alden } \\
\text { Santa Fe } \\
\text { Mickelson } \\
\text { Silver City } \\
\text { P. M. Singer } \\
\text { State College } \\
\text { P. K. Rees }\end{array}$ & $\begin{array}{l}\text { m Johns- } \\
\text { Berry } \\
\text { y } \\
\text { ord } \\
\text { stitute } \\
\text { s } \\
\text { Smith } \\
\text { Thompson } \\
\text { eph's Col- } \\
\text { or Women } \\
\text { is Xavier } \\
\text { ele } \\
\text { lan } \\
\text { lle College } \\
\text { Imelda } \\
\text { ity of Buf- } \\
\text { an } \\
\text { igue } \\
\text { Ott } \\
\text { l } \\
\text { Brown } \\
\text { th } \\
\text { on } \\
\text { nbacher } \\
\text { Point } \\
\text { enderson } \\
\text { Wright } \\
\text { City } \\
\text { wden } \\
\text { s } \\
\text { s }\end{array}$ \\
\hline
\end{tabular}




\begin{tabular}{|c|c|c|c|}
\hline & $\begin{array}{l}\text { Moir } \\
\text { Murphy } \\
\text { Neuman } \\
\text { Otten } \\
\text { Parsons } \\
\text { E. A. Porter } \\
\text { Preston } \\
\text { C. A. Richmond } \\
\text { Robbins } \\
\text { Roosevelt } \\
\text { L. G. Simon } \\
\text { D. E. Smith } \\
\text { Tanzola } \\
\text { Warner } \\
\text { W. Weaver } \\
\text { A. W. Whitney } \\
\text { E. C. Williams } \\
\text { W. J. Willis } \\
\text { American Mathe- } \\
\text { matical Society } \\
\text { E. M. Hull } \\
\text { Odoms } \\
\text { American } \\
\text { phone and Tele- } \\
\text { graph Company } \\
\text { Bagnall } \\
\text { Belcher } \\
\text { J. R. Carson } \\
\text { R. M. Foster } \\
\text { Halbert } \\
\text { Hoyt } \\
\text { Mead } \\
\text { Bell Telephone } \\
\text { Laboratories } \\
\text { Bode } \\
\text { Dietzold } \\
\text { Fry } \\
\text { Gray } \\
\text { P. C. Jones } \\
\text { Kammerer } \\
\text { MacColl } \\
\text { Molina } \\
\text { Olmstead } \\
\text { Riordan } \\
\text { Schelkunoff } \\
\text { Sunde } \\
\text { Vaage } \\
\text { Wise } \\
\text { Zobel } \\
\text { College of the City } \\
\text { of New York } \\
\text { Boeker } \\
\text { Carrie } \\
\text { Echols } \\
\text { B. P. Gill } \\
\text { C. C. Grove } \\
\text { Hubert } \\
\text { Ingram } \\
\text { Linehan } \\
\text { Nathan } \\
\text { Philip } \\
\text { Post } \\
\text { F. G. Reynolds } \\
\text { S. L. Robinson }\end{array}$ & 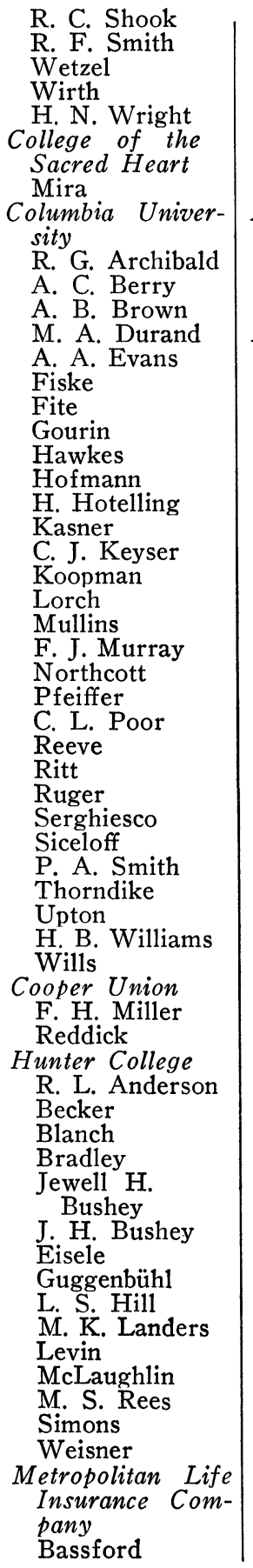 & 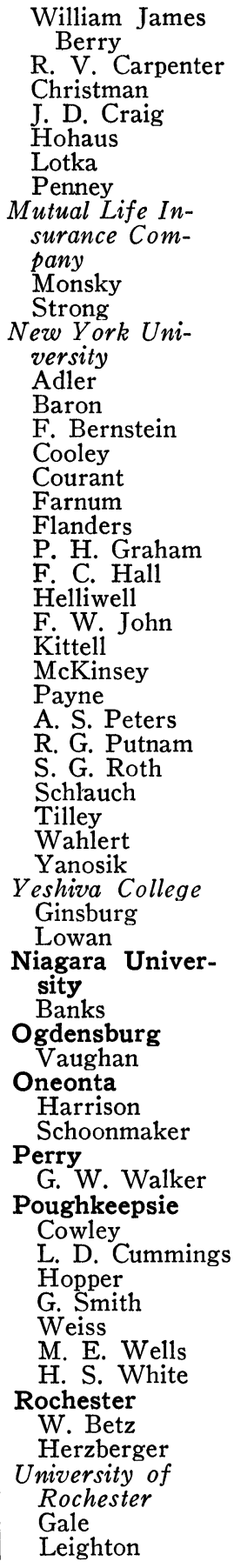 \\
\hline
\end{tabular}




\begin{tabular}{|c|c|c|c|}
\hline $\begin{array}{l}\mathrm{B} \\
\mathrm{F} \\
\mathrm{A} \\
\mathrm{G} \\
\mathrm{D} \\
\mathrm{A} \\
\mathrm{U} \\
\mathrm{V}\end{array}$ & $\begin{array}{l}\text { Durham } \\
\text { Carlitz } \\
\text { F. G. Dressel } \\
\text { W. W. Elliott } \\
\text { Gergen } \\
\text { Greenwood } \\
\text { Hickson } \\
\text { E. R. C. Miles } \\
\text { Patterson } \\
\text { Roberts } \\
\text { J. M. Thomas } \\
\text { Greensboro } \\
\text { Daniels } \\
\text { Pegram } \\
\text { Greenville } \\
\text { ReBarker } \\
\text { Maxton } \\
\text { Key } \\
\text { Raleigh } \\
\text { Bullock } \\
\text { Cell } \\
\text { J. M. Clarkson } \\
\text { Levine } \\
\text { H. V. Park } \\
\text { Salisbury } \\
\text { Dearborn } \\
\text { Statesville } \\
\text { R. W. Stokes } \\
\text { NORTH } \\
\text { DAKOTA } \\
\text { Fargo } \\
\text { I. W. Smith } \\
\text { OHIO } \\
\text { Alliance } \\
\text { Hildner } \\
\text { Ashland } \\
\text { Rinehart } \\
\text { Athens } \\
\text { Marquis } \\
\text { F. W. Reed } \\
\text { Starcher } \\
\text { Bluffton } \\
\text { Hirschler } \\
\text { Bowling Green } \\
\text { Ogg } \\
\text { Overman } \\
\text { Cincinnati } \\
\text { University of Cin- } \\
\text { cinnati } \\
\text { Barnett } \\
\text { Brand } \\
\text { Farnau } \\
\text { Hancock } \\
\text { Kersten } \\
\text { Lubin } \\
\text { Mendel } \\
\text { Merriman } \\
\text { H. L. Miller } \\
\text { C. N. Moore } \\
\text { P. M. Pepper } \\
\text { Szász } \\
\text { Yowell } \\
\text { Dato }\end{array}$ & 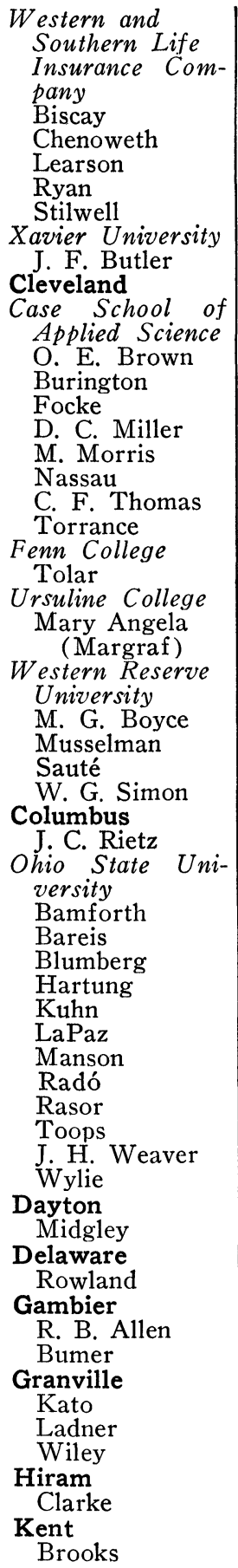 & $\begin{array}{l}\text { m- } \\
\text { s } \\
\text { on }\end{array}$ \\
\hline
\end{tabular}




\begin{tabular}{|c|c|c|c|}
\hline $\begin{array}{l}\text { Allentown } \\
\text { Cedar Crest Col- } \\
\text { lege for Wom- } \\
\text { en } \\
\text { Hallett } \\
\text { Muhlenberg Col- } \\
\text { lege } \\
\text { Deck } \\
\text { Koehler } \\
\text { Annville } \\
\text { P. S. Wagner } \\
\text { Bethlehem } \\
\text { Beale } \\
\text { S. S. Cairns } \\
\text { R. N. Cobb } \\
\text { Cutler } \\
\text { Fort } \\
\text { Lamson } \\
\text { Latshaw } \\
\text { D. H. Lehmer } \\
\text { Raynor } \\
\text { C. A. Shook } \\
\text { Smail } \\
\text { Vanderslice } \\
\text { Bryn Mawr } \\
\text { Comegys } \\
\text { T. S. Peterson } \\
\text { Steinmetz } \\
\text { Bryn Marer Col- } \\
\text { lege } \\
\text { Hedlund } \\
\text { Lehr } \\
\text { Tuller } \\
\text { A. P. Wheeler } \\
\text { California } \\
\text { Bernstorf } \\
\text { Carlisle } \\
\text { F. Ayres } \\
\text { Landis } \\
\text { Chambersburg } \\
\text { Davison } \\
\text { R. F. Johnson } \\
\text { Weeks } \\
\text { Chester } \\
\text { F. G. Williams } \\
\text { Clarion } \\
\text { C. E. Carey } \\
\text { Collegeville } \\
\text { Clawson } \\
\text { Clate }\end{array}$ & 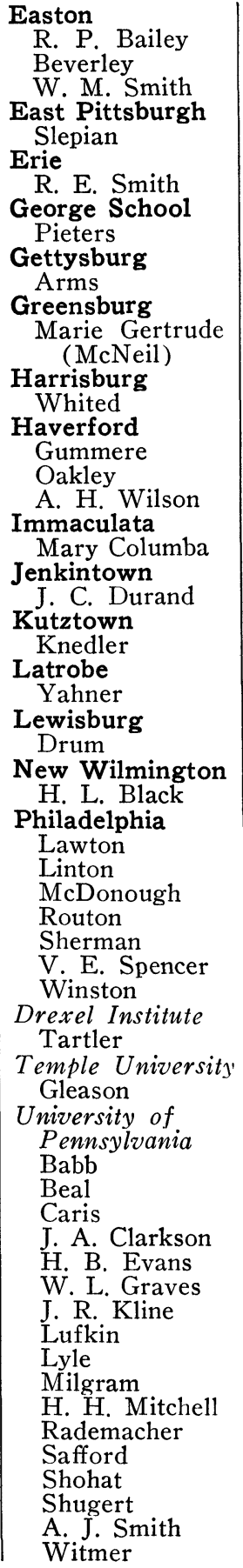 & 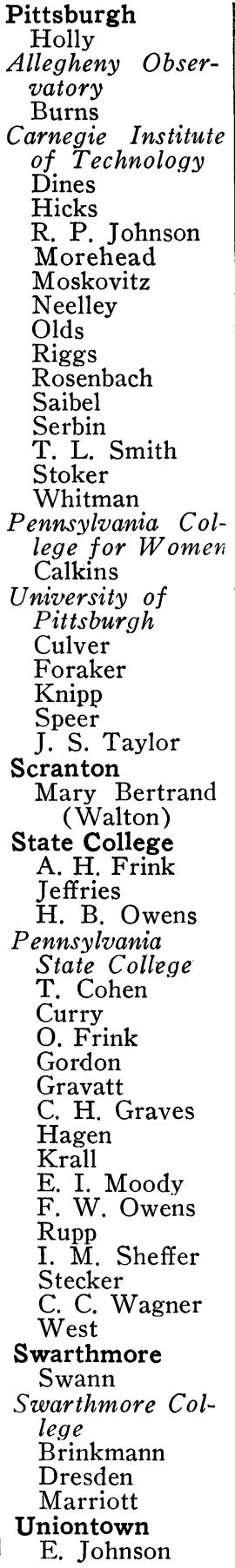 & $\begin{array}{l}\text { RHODE } \\
\text { ISLAND } \\
\text { Providence } \\
\text { R. B. Adams } \\
\text { Brown University } \\
\text { C. R. Adams } \\
\text { R. C. Archibald } \\
\text { A. A. Bennett } \\
\text { D. L. Bernstein } \\
\text { Carlen } \\
\text { Coon } \\
\text { C. H. Currier } \\
\text { Eberhart } \\
\text { Frame } \\
\text { Gilman } \\
\text { Lindsay } \\
\text { H. P. Manning } \\
\text { Middleswart } \\
\text { Rawhouser } \\
\text { R. G. D. Rich- } \\
\text { ardson } \\
\text { R. M. Robinson } \\
\text { Royall } \\
\text { C. H. Smiley } \\
\text { Tamarkin } \\
\text { Tukey } \\
\text { West Warwick } \\
\text { Brant } \\
\text { SOUTH } \\
\text { CAROLINA } \\
\text { Charleston } \\
\text { J. Anderson } \\
\text { Dye } \\
\text { Clinton } \\
\text { H. E. Spencer } \\
\text { Columbia } \\
\text { J. B. Coleman } \\
\text { J. B. Jackson }\end{array}$ \\
\hline
\end{tabular}




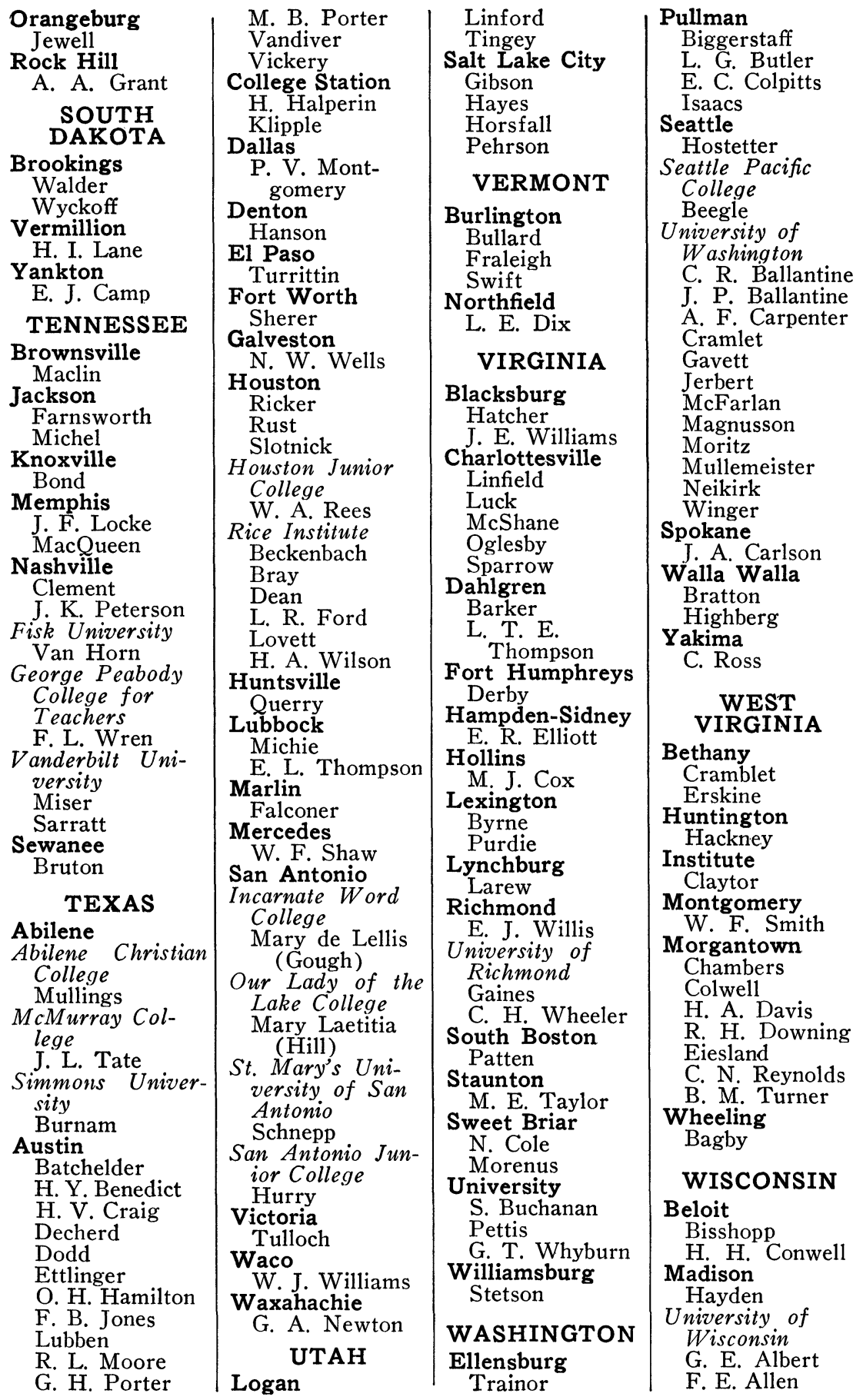


T. Bennett

Breit

Erickson

H. P. Evans

I. G. Foster

V. C. Harris

W. W. Hart

Ingraham

Kiokemeister

Kleene

Langer

MacDuffee

March

May

Scheffé

Schwid

Slichter

E. S. Sokolnikoff I. S. Sokolnikoff Trump

E. B. Van Vleck O. L. Wheeler

M. C. Wolf

Milwaukee

Bardell

P. H. Evans

Marquette University

Pettit

Wilczewski

Milwaukee-

Downer College

Beckwith

State Teachers

College

Knight

Norris

University of

Wisconsin at

Milwaukee

Battig

Marden

Nordhaus

Parkinson

W. E. Roth

Vass

L. A. Wolf

Oshkosh

Beenken

I. Price

River Falls

Eide

Superior

C. W. Smith

West De Pere

DeCleene

Williams Bay

Collins

Wisconsin Rapids

McMillan

WYOMING

Laramie

Bellamy
CANADA

Alberta

Edmonton

J. W. Campbell

A. J. Cook

Sheldon

British Columbia

Vancouver

D. Buchanan

Gage

Nowlan

Manitoba

Winnipeg

N. R. Wilson

New Brunswick

Sackville

W. H. McEwen

. Nova Scotia

Halifax

Adshead

Wolfville

H. F. Archibald

Jeffery

Ontario

Hamilton

Findlay

Kingston

Gummer

Matheson

N. Miller

Watts

London

Kingston

Wiancko

Ottawa

G. V. Brady

A. W. Turner

Toronto

Wolfenden

University of Toronto

Beatty

Brauer

Burk

DeLury

Gilchrist

S. H. Gould

Krieger

Pounder

G. D. Robinson

Rosebrugh

Synge

Waddell

W. J. Webber

\section{Quebec}

Lennoxville

A. V. Richardson
Montreal

C. Craig

MacLean

Pall

A. N. Shaw

C. T. Sullivan

H. Tate

W. L. G. Williams

Quebec

Tremblay

Saskatchewan

Saskatoon

Ling

ARGENTINA

Buenos Aires

Baidaff

AUSTRIA

Vienna

Gödel

AUSTRALIA

Adelaide

Wilton

Perth

Weatherburn

Sydney

Thorne

CANAL ZONE

Quarry Heights

Sinkov

CHINA

Amoy

Chang

Canton

Woo

Peiping

National University of Peiping

Kiang

T sing Hua University

Tseng

Tientsin

Chiang

CUBA

Havana

Corral-Alemán

DENMARK

Copenhagen

Royal Technical College

Jessen

University of Copenhagen

Bohr

Nörlund
FRANCE

Paris

Fréchet

Potron

\section{GERMANY}

Berlin

Bieberbach

Freiburg i. Br.

Bolza

Giessen

Geppert

Hamburg

Blaschke

Jena

König

Leipzig

AckermannTeubner

Deuring

\section{GREAT}

BRITAIN

England

Birmingham

Watson

Bristol

Fraser

Cambridge

Macaulay

University of Cam-

bridge

Carslaw

Collingwood

G. H. Hardy

Larmor

Ramsey

H. W. Richmond

Taussky

Leeds

W. P. Milne

London

Brill

Tavani

Western

University of Lon-

don

Bosanquet

Hilton

Manchester Mordell

Oxford

Dixon

E. B. Elliott

Frecheville

A. E. H. Love

Whitehead

Stocksfield-onTyne

Hardcastle

Suffolk

Curjel 


Scotland
Dundee
Copson
Edinburgh
Jaramillo
Whittaker
Wales
Bangor
Berwick
Cardiff
Livens
Swansea
A. R. Richardson
GREECE
Athens
Zervos
HUNGARY
Budapest
Fejér
C. Jordan
Szeged
de Kerékjártó
INDIA
Aligarh
Shah
Annamalainagar
Rao
Bangalore
Iyengar
Bareilly
Bhattacharyya

\begin{tabular}{|l} 
Bombay \\
Dalal \\
Dacca \\
Vijayaraghavan \\
Delhi \\
Seth \\
Lucknow \\
Paranjpye \\
Madras \\
Ananda-Rau \\
$\quad$ IRAQ \\
Baghdad \\
Merrick \\
IRELAND \\
Dublin \\
McConnell \\
Rowe \\
ITALY \\
Palermo \\
Mineo \\
Pisa \\
Ricci \\
Tonelli \\
Rome \\
Levi-Civita \\
Volterra \\
Turin \\
Fano \\
JAPAN \\
Chibaken \\
Hirayama \\
\end{tabular}

Sapporo
Kawaguchi
Sendai
Kubota
Okada
Takasu
Tokyo
Kuniyeda
Yoshida
Yamagata
Yanagihara
NEW
ZEALAND
Auckland
Forder
PALESTINE
Jerusalem
Levitzki
PANAMA
Panama City
Linares
PERU
Lima
de Losada y Puga
POLAND
Lwów
Ulam
Warsaw
Dickstein

\begin{tabular}{|c} 
SIAM \\
Bangkok \\
Hadlock \\
STRAITS SET- \\
TLEMENTS \\
Singapore \\
Oppenheim \\
SWEDEN \\
Stockholm \\
Oseen \\
SWITZER- \\
LAND \\
Zurich \\
Gut \\
TURKEY \\
Istanbul \\
Uzdilek \\
UNION OF \\
SOVIET \\
SOCIALIST \\
REPUBLICS \\
Kieff \\
Kryloff \\
Leningrad \\
Smouroff \\
VENEZUELA \\
Caripito \\
C. H. Dix
\end{tabular}

